\title{
Rheology in Polymer/Clay Nanocomposites: Mesoscale Structure Development and Soft Glassy Dynamics
}

\section{Masami Okamoto}

\section{CONTENTS}

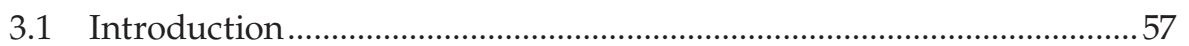

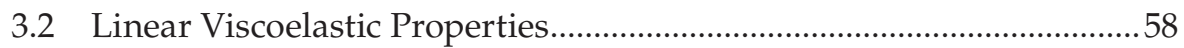

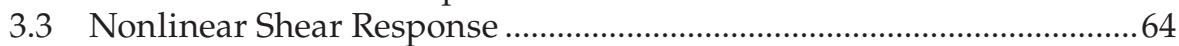

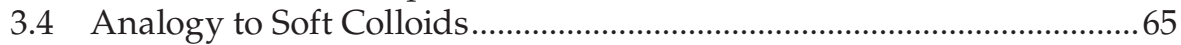

3.5 Reversibility of Network Formation Process ............................................66

3.6 Alignment of Silicate Layers in Networks ........................................... 70

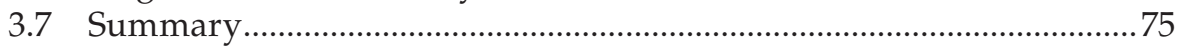

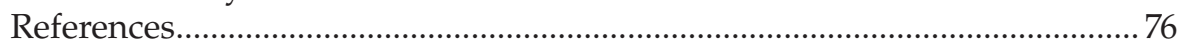

\subsection{Introduction}

Over the last few years, the utility of inorganic nanoscale particles as filler to enhance the polymer performance has been established. Of particular interest is recently developed nanocomposite technology consisting of a polymer and organically modified layered filler (organo-clay), because they often exhibit remarkably improved mechanical and various other materials properties as compared with those of virgin polymer or conventional composite (micro-/macrocomposites) [1-6]. These concurrent property improvements are well beyond what can generally be achieved through the micro-/macrocomposites preparation.

The synthetic strategy and molecular design was first explored by Toyota group with nylon 6 as the matrix polymer [7]. This new class of material is now being introduced in structural applications, such as gas barrier film and other load-bearing applications [5]. Polymer/clay nanocomposites (PCNs) and their self-assembly behaviors have recently been approached to produce nanoscale polymeric materials [1-6]. Additionally, these nanocomposites 
have been proposed as model systems to examine polymer structure and dynamics in confined environments [8-10].

In order to understand the processability of these materials (i.e., the final stage of any polymeric material), one must understand the detailed rheological behavior of these materials in the molten state. Understanding the rheological properties of PCN melts is not only important in gaining a fundamental knowledge of the processability but is also helpful in understanding the structure-property relationships in these materials. Although rheological measurement is an indirect probe, it is a well-established approach to probe the interaction between nanofiller and polymer matrix and the time-dependent structure development. In addition, more clear nanoscale and mesoscale structure development of the systems could be provided when combined with X-ray/light-scattering experiments and electron microscopy.

The original mesoscale structure in PCNs consists of randomly oriented exfoliated layers or tactoids of layers. This randomly distributed nanofiller forms a "organo-clay network" structure that is mediated by polymer chains and organo-clay-organo-clay interactions, responsible for the linear viscoelastic response observed in PCN melts. This mesostructure, which is intrinsically in a disordered metastable state and out of equilibrium, and offers an apt analogy to soft colloidal glasses and gels, was extensively discussed [11,13-27].

In this chapter, we survey mesostructure development in PCN melts with a primary focus on flow behavior and an analogy to soft glassy dynamics [12].

\subsection{Linear Viscoelastic Properties}

Dynamic oscillatory shear measurements of polymeric materials are generally performed by applying a time-dependent strain of $\gamma(t)=\gamma_{o} \sin (\omega t)$, and the resultant shear stress is $\sigma(t)=\gamma_{0}\left[G^{\prime} \sin (\omega t)+G^{\prime \prime} \cos (\omega t)\right]$, with $G^{\prime}$ and $G^{\prime \prime}$ being the storage and loss modulus, respectively.

Generally, the rheology of polymer melts strongly depends on the temperature at which measurement is carried out. It is well known that for thermorheological simplicity, isotherms of storage modulus $\left(G^{\prime}(\omega)\right)$, loss modulus $\left(G^{\prime \prime}(\omega)\right)$, and complex viscosity $\left(\left|\eta^{*}\right|(\omega)\right)$ can be superimposed by horizontal shifts along the frequency axis:

$$
\begin{aligned}
& b_{\mathrm{T}} G^{\prime}\left(a_{\mathrm{T}} \omega, T_{\text {ref }}\right)=b_{\mathrm{T}} G^{\prime}(\omega, T) \\
& b_{\mathrm{T}} G^{\prime \prime}\left(a_{\mathrm{T}} \omega, T_{\text {ref }}\right)=b_{\mathrm{T}} G^{\prime \prime}(\omega, T) \\
& \left|\eta^{*}\right|\left(a_{\mathrm{T}} \omega, T_{\text {ref }}\right)=\left|\eta^{*}\right|(\omega, T)
\end{aligned}
$$


where $a_{\mathrm{T}}$ and $b_{\mathrm{T}}$ are the frequency and vertical shift factors, respectively; and $T_{\text {ref }}$ is the reference temperature. All isotherms measured for pure polymer and for various PCNs can be superimposed along the frequency axis.

In the case of polymer samples, it is expected, at the temperatures and frequencies at which the rheological measurements were carried out, that the polymer chains should be fully relaxed and exhibit characteristic homopolymer-like terminal flow behavior (i.e., curves can be expressed by a power law of $G^{\prime}(\omega) \propto \omega^{2}$ and $\left.G^{\prime \prime}(\omega) \propto \omega\right)$.

The rheological properties of in situ polymerized nanocomposites with end-tethered polymer chains were first described by Krisnamoorti and Giannelis [13]. The flow behavior of poly(_-caprolactone) (PCL)- and nylon 6-based nanocomposites differed extremely from that of the corresponding neat matrices, whereas the thermorheological properties of the nanocomposites were entirely determined by that behavior of matrices [13]. The slope of $G^{\prime}(\omega)$ and $G^{\prime \prime}(\omega)$ versus the $a_{\mathrm{T}} \omega$ is much smaller than 2 and 1 , respectively. Values of 2 and 1 are expected for linear monodispersed polymer melts and are a large deviation, and especially in the presence of a very small amount of layered silicate, loading may be due to the formation of network structure in the molten state. However, such nanocomposites based on the in situ polymerization technique exhibit fairly broad molar mass distribution of the polymer matrix, which hides the structurally relevant information and impedes interpretation of the results.

To date, the melt state linear dynamic oscillatory shear properties of various kinds of PCNs have been examined for a wide range of polymer matrices, including nylon 6 with various matrix molecular weights [14], PS [15], polystyrene (PS)-polyisoprene (PI) block copolymers [16,17], PCL [18], polypropylene (PP) [11,19-22], polylactide (PLA) [23,24], and poly(butylene succinate) (PBS) $[25,26]$. In the linear viscoelastic regime, a big change in the terminal (low-frequency) region from a liquid-like response to a solidlike response was observed for all PCNs $\left(G^{\prime}(\omega) \sim G^{\prime \prime}(\omega) \propto \omega^{0}\right)$, ascribed to the formation of a volume spanning mesoscale organo-clay network above the mechanical percolation threshold [22]. The terminal rheology is sensitive to organo-clay loading and the extent of exfoliation/intercalation in the polymer matrix.

A typical example of the linear dynamic viscoelastic master curves for the neat polylactide (PLA) and various PLA-based nanocomposites (PLACNs) with different organo-clay loading [24] is shown in Figure 3.1. The linear dynamic viscoelastic master curves were generated by applying the timetemperature superposition principle and shifted to a common temperature $T_{\text {ref }}$ using both frequency shift factor $a_{\mathrm{T}}$ and modulus shift factor $b_{\mathrm{T}}$. The moduli of the PLACNs increase with increasing clay loading at all frequencies $\omega$. At high $\omega s$, the qualitative behavior of $G^{\prime}(\omega)$ and $G^{\prime \prime}(\omega)$ is essentially the same and is unaffected by frequencies. However, at low frequencies, $G^{\prime}(\omega)$ and $G^{\prime}(\omega)$ increase monotonically with increasing organo-clay content. In the low-frequency region, the curves can be expressed by power law of $G^{\prime}(\omega) \propto \omega^{2}$ 


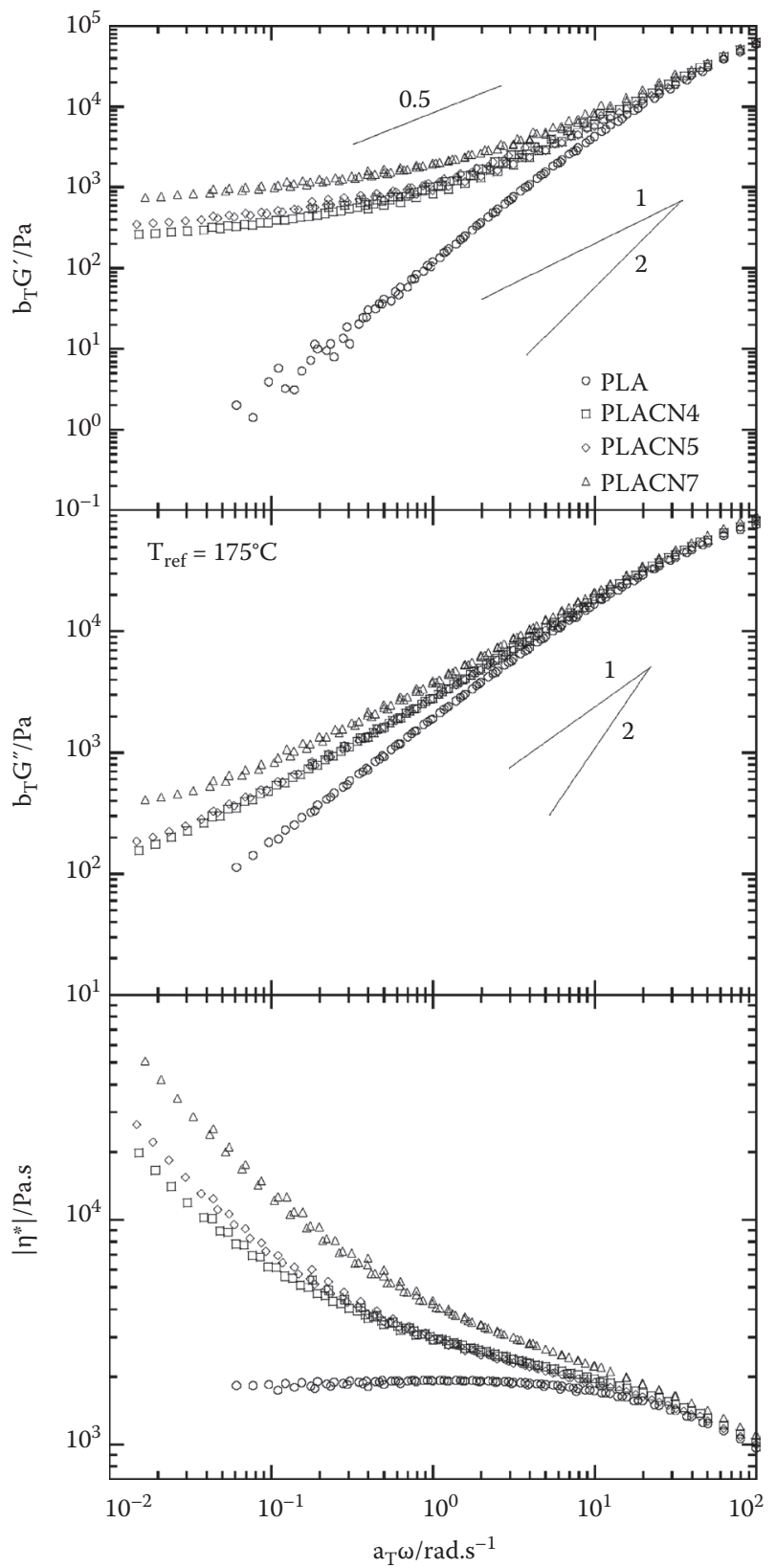

FIGURE 3.1

Reduced frequency dependence of storage modulus, loss modulus, and complex viscosity of neat polylactide (PLA) and various PLA-based nanocomposites (PLACNs) under dynamic oscillatory shear measurement. (Reprinted from Sinha Ray S, Yamada K, Okamoto M, Ueda K, Polymer 44, 6631. Copyright 2003 Elsevier Science. With permission.) 
TABLE 3.1

Terminal Slopes of $\mathrm{G}^{\prime}$ and $\mathrm{G}^{\prime \prime}$ versus $a_{\mathrm{T}} \omega$ for Polylactide (PLA) and Various PLA-Based Nanocomposites (PLACNs)

\begin{tabular}{lcccc}
\hline System & Organo-Clay/wt $\%$ & $G^{\prime}$ & $G^{\prime \prime}$ & $E_{\mathrm{a}} \mathbf{a} / \mathbf{k J m o l}^{-1}$ \\
\hline PLA & 0 & 1.6 & 0.9 & $170 \pm 5$ \\
PLACN4 & 4.0 & 0.2 & 0.5 & $225 \pm 10$ \\
PLACN5 & 5.0 & 0.18 & 0.4 & $225 \pm 5$ \\
PLACN7 & 7.0 & 0.17 & 0.32 & $230 \pm 8$ \\
\hline
\end{tabular}

a Flow activation energy obtained from an Arrhenius fit of master curves.

Source: Reprinted from Sinha Ray S, Yamada K, Okamoto M, Ueda K, Polymer 44, 6631, (C) 2003, Elsevier Science. (With permission.)

and $G^{\prime \prime}(\omega) \propto \omega$ for neat PLA, suggesting this is similar to those of the narrow molecular weight $\left(M_{\mathrm{w}}\right)$ distribution homopolymer melts. On the other hand, for $a_{\mathrm{T}} \omega<5$ rad. $\mathrm{s}^{-1}$, the viscoelastic response (particularly $G^{\prime}(\omega)$ ) for all the nanocomposites displays significantly diminished frequency dependence as compared with the matrices. In fact, for all PLACNs, $G^{\prime}(\omega)$ becomes nearly independent at low $a_{\mathrm{T}} \omega$ and exceeds $G^{\prime \prime}(\omega)$, characteristic of materials exhibiting a pseudo-solid-like behavior. The terminal zone slopes values of both neat PLA and PLACNs are estimated at the lower $a_{\mathrm{T}} \omega$ region $\left(<10 \mathrm{rad}_{\mathrm{s}} \mathrm{s}^{-1}\right)$ and are presented in Table 3.1. The lower slope values and the higher absolute values of the dynamic moduli indicate the formation of an "organo-clay network" structure in the PLACNs in the molten state [16,24]. This mesoscale structure is partially supported by transmission electron microscopic (TEM) observation [24]. The correlation length between clay particles ( $\left.\xi_{\text {clay }}\right)$ is smaller than the average value of the particle length $\left(L_{\text {clay }}\right)$ for PLACNs, suggesting the formation of highly geometric constraints. A plausible explanation and its model are presented by Ren et al. [16] (Figure 3.2). The individual or stacked silicate layers are incapable of freely rotating; hence, by imposing small $\omega$, the relaxation of the structure is prevented almost completely. This type of prevented relaxation leads to the presence of the volume-spanning mesoscale network. The effective volume occupied by the clay platelets is much larger than may be calculated from their volume alone. At the same time, the pseudo-solid-like behavior is observed in all PCNs, despite the fact that the thermodynamic interaction between those polymers and organo-clay is quite different [11,13-27]. In addition, the correlation between rheological response and the confinement of polymer chains into silicate nanogalleries is reported [28]. The confinement is directly concerned with rheological properties and enhanced modulus.

The temperature dependence frequency shift factor $\left(a_{\mathrm{T}}\right.$, Williams-LandelFerry (WLF) type [29]) used to generate the master curves shown in Figure 3.1 is shown in Figure 3.3. The dependence of the frequency shift factors on the 


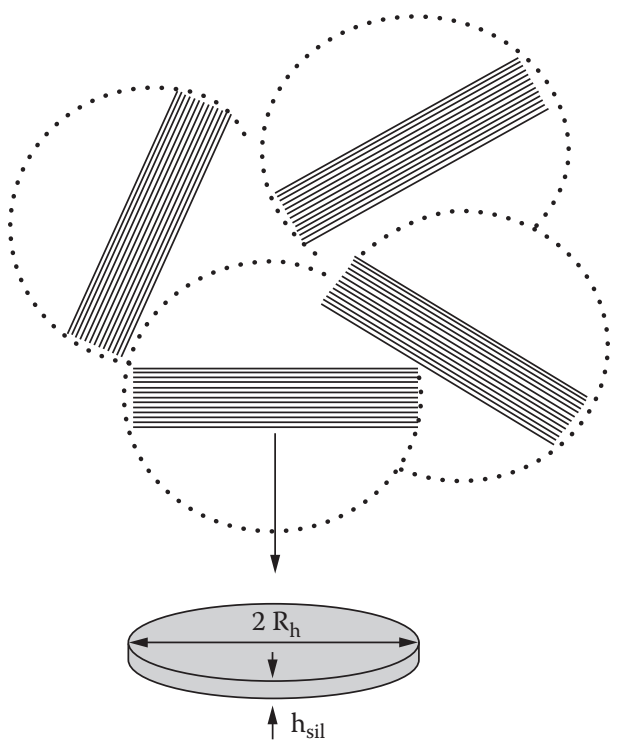

FIGURE 3.2

Percolation of hydrodynamic volumes of silicate layers at low concentration. Stacked silicate layers and their interaction with each other, resulting in complete relaxation of the nanocomposite melts. (Reprinted from Ren J, Silva AS, Krishnamoorti R, Macromolecules 33, 3739. Copyright 2000 American Chemical Society. With permission.)

silicate loading suggests that the temperature-dependent relaxation process observed in the viscoelastic measurements is somehow affected by the presence of the silicate layers $(225 \mathrm{~kJ} / \mathrm{mol}$ for PLACN4 and $170 \mathrm{~kJ} / \mathrm{mol}$ for neat PLA, see Table 3.1). In the case of nylon 6-based nanocomposites, where the hydrogen bonding on the already formed hydrogen-bonded molecule to the silicate surface [30], the system exhibits a large flow activation energy [AU: Sense OK? almost one order higher in magnitude compared with that of neat nylon 6 (450 kJ/mol and $1900 \mathrm{~kJ} / \mathrm{mol}$ for nylon 6-based nanocomposites with $1.6 \%$ and $3.7 \%$ clay loading, respectively, and $350 \mathrm{~kJ} / \mathrm{mol}$ for neat nylon 6 ) (see Figure 3.10) [31].

The shift factor $b_{\mathrm{T}}$ shows large deviation from a simple density effect, and it would be expected that the values would not vary far from unity $\left(b_{\mathrm{T}}=\rho T / \rho_{\text {ref }} T_{\text {ref }}\right.$, where $\rho$ and $\rho_{\mathrm{f}}$ are the densities at $T$ and $T_{\text {ref }}$, respectively) [29]. One possible explanation is a network structural change occurring in PLACNs during measurement (shear process). The reconstituting of the organo-clay network probably supports for PCN melts under weak shear flow (terminal zone), thereby leading to the increase in the absolute values of $G^{\prime}(\omega)$ and $G^{\prime \prime}(\omega)$.

The subsequent reconstitution of organo-clay domains into a mesoscale network is also argued by simple Brownian motion, where the rotary diffusivity $\left(D_{\mathrm{r} 0}\right)$ of a circular disk of diameter $(d)\left(=L_{\text {clay }} / 2\right)$ is $[32,33]$ 


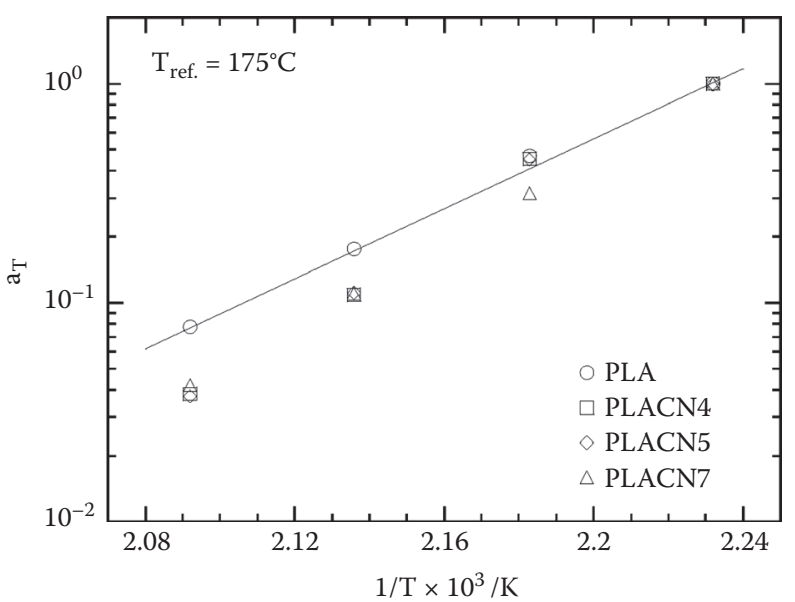

(a)

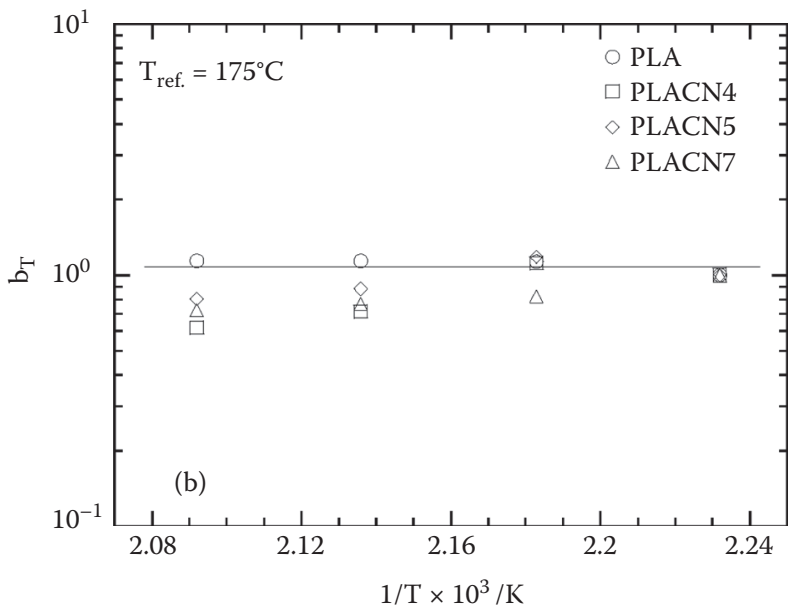

(b)

FIGURE 3.3

(a) Frequency shift factors $a_{\mathrm{T}}$ and (b) modulus shift factor $b_{\mathrm{T}}$ as a function of temperature. (Reprinted from Sinha Ray S, Yamada K, Okamoto M, Ueda K, Polymer 44, 6631.Copyright 2003, Elsevier Science. With permission.)

$$
D_{r 0}=\frac{3 k_{B} T}{4 \eta_{0} d^{3}}
$$

where $\eta_{0}$ is the polymer matrix viscosity, $k_{\mathrm{B}}$ is the Boltzmann constant, and $T$ is the temperature.

The rotational relaxation time $\left(t_{\mathrm{D}}\right)$ is given by 


$$
t_{D} \sim \frac{(\pi / 2)^{2}}{D_{r 0}}
$$

A quarter period is provided to complete rotational relaxation. This timescale is useful to discuss the reconstituting of the network via the rotation of the organo-clays as compared with an experimental timescale. For PLACN4, the estimated timescale for rotational Brownian motion is about $2 \times 10^{3} \mathrm{~s}$ at $175^{\circ} \mathrm{C}$. This value is larger than the experimental timescale $\left(1 / a_{\mathrm{T}} \omega \sim 10^{2} \mathrm{~s}\right)$, suggesting the major driving force for the structural evolution is not simple Brownian relaxation of the organo-clays. The extensive argument of the reorganization of organo-clay domains in light of the rotational Brownian motion has been presented in several papers [11,19,33,34].

However, in many intercalated nanocomposites (i.e., complete exfoliation is not feasible), the organo-clay domains consist of the penetrated polymer chains into nanogalleries and stacked silicate layers [35]. Irrespective of the intercalated nanocomposite structure, the opposite conclusion was also reported with respect to the timescale between Brownian relaxation and experimental relaxation [33]. In this regard, we have to clarify the mesoscale network structure. For this reason, the stress recovery following prolonged large-amplitude oscillatory shear (LAOS) $[19,33]$ and the stress response upon start-up of steady shear or small-amplitude oscillatory shear (SAOS) [11,22] were examined to contrast the aging behavior of as-processed (unsheared) and presheared (leading to parallel alignment of the silicate layers) nanocomposites.

\subsection{Nonlinear Shear Response}

The start-up of shear and SAOS experiments offer insight into mesoscale network structural changes.

Solomon et al. conducted nonlinear reversing shear flow experiments to study the disorientation kinetics of flow-aligned organo-clay domains in a PP matrix during the annealing period between deformations. The transient stress in start-up of steady shear scaled with the applied strain (not shear rate) (Figure 3.4) [19]. This transient nonlinear rheology has non-Brownian style. They showed the disorientation kinetics faster than that predicted by Brownian motion and the constant WLF shift factor for the PP matrix and nanocomposites, and they concluded that the strong attraction between the silicate layers led to the rapid disorientation upon cessation of shear [19]. They suggested that the stacked silicate layers into larger organo-clay domains assemble into a heterogeneous organo-clay network as a result of attractive interaction (van der Waals force). The organo-clay network formed under quiescent conditions is easily perturbed by deformation. Upon the cessation 


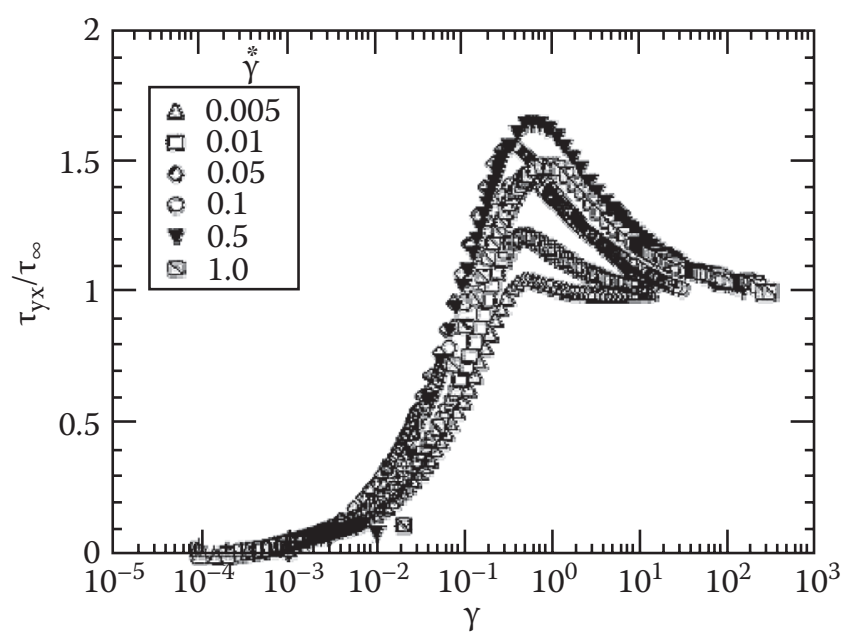

\section{FIGURE 3.4}

Onset of stress overshoot in start-up of steady shear depends on the strain applied to the polypropylene (PP)-based nanocomposite at $180^{\circ} \mathrm{C}$. (Reprinted from Solomon MJ, Almusallam AS, Seefeld KF, Somwangthanaroj S, Varadan P, Macromolecules 34, 1864.Copyright 2001 American Chemical Society. With permission.)

of flow, attractive interparticle interactions promote reconstitution of the network. The reconstituting network, which more completely re-forms as the rest time increases, is the initial state upon which deformation is oriented and ruptured, thereby giving rise to an overshoot in the stress during the flow reversal experiments [19].

For viscosity overshoot, Treece et al. reported that the flow ruptures the organo-clay network in the as-processed material and flow-aligns its domains to a degree that depends on the magnitude of the deformation [11]. The morphology in both as-processed and presheared samples changes significantly during annealing. This result supports the large increases in the magnitude of the viscosity overshoot. The strong dependence on annealing time for the as-processed sample clearly indicates that it is out of equilibrium $[11,28]$. This behavior is an "aging" phenomenon characteristic of systems (i.e., microgel pastes, nematic polymer, aqueous hectrite-type clay [Laponite] dispersion). Those are classified as soft colloidal glasses and are far from equilibrium (Figure 3.5) [36].

\subsection{Analogy to Soft Colloids}

Ren et al. [33] discussed the analogy to the dynamics of soft colloidal glasses, exhibiting yield stress, thixotropy, and slow stress recovery under 


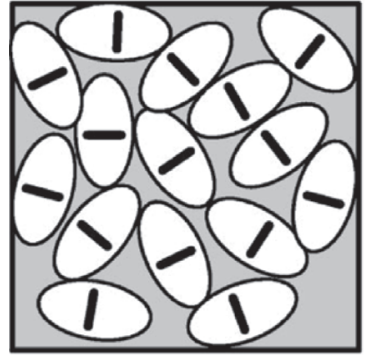

(a)

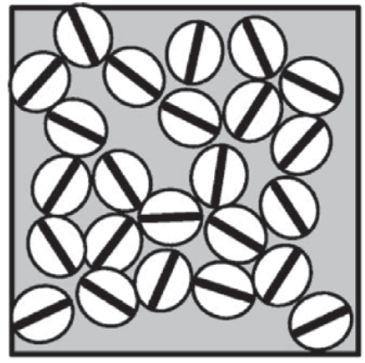

(b)

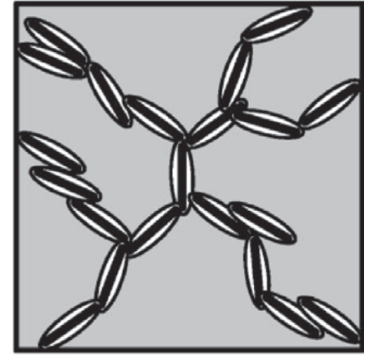

(c)

FIGURE 3.5

(a) Repulsive colloidal glass, (b) attractive glass, and (c) gel. Each thick line represents a Laponite disk, and a white ellipsoid indicates the range of electrostatic repulsions. For (a), long-range electrostatic repulsions dominate. In (b), attractive interactions affect the spatial distribution, but repulsive interactions still play the predominant role in the slow dynamics of the system. In (c), attractive interactions play a dominant role; a percolated network forms, which gives the system its elasticity. (Reprinted from Tanaka H, Meunier J, Bonn D, Phys Rev. E 69, 031404. Copyright 2004 American Physical Society. With permission.)

deformation [12,36]. A glass is homogenous on interparticle structure with elasticity derived from the caging effect, which is characterized by long-range electrostatic repulsion and short-range attraction [36]. In the colloidal solid, the storage modulus and complex viscosity show logarithmic dependence time, like PCN melts. For example, in an aqueous Laponite dispersion, the structure reorganization responsible for the rheological behavior depends upon the ionic strength of the dispersion, which balances the attractive and repulsive forces between clay domains.

On the other hand, a gel also shows the soft glassy dynamics, which is associated with metastable, structural heterogeneity on mesoscale length, caused by a percolation infinite (volume spanning) network. PCNs have a structural hierarchy without long-range repulsive interaction, leaving short-range attractive forces (van der Waals forces) between polymer and clay because of the surface modification of clay by cationic intercalant. For this reason, PCN melts were classified as attractive colloidal gels [11]. This picture supports other experimental results on transient start-up viscosity under shear flow $[22,33]$.

\subsection{Reversibility of Network Formation Process}

The central discussion in PCN melts is structure with elasticity. The idea is an organo-clay network with a few weight percent (wt\%). An important question is how long the building of the solid-like network continues during annealing (i.e., reversibility of the network formation process). 
In an aqueous dilute suspension of smectite swelling clays such as hectorite and montmorillonite, the existence of a long-range structural correlation $(\sim 100 \mathrm{~nm})$ was proposed via an in situ small-angle neutron scattering (SANS) experiment of the smectite suspension under the quiescent state [37]. This is a worthy hypothesis for a new interpretation of network formation and complex rheological properties. Such magnitude of the structural length in the clay suspensions is suitable for a Rayleigh scattering experiment on the basis of the different polarizabilities between clay particles and medium.

Okamoto et al. constructed a rheo-optical device, small-angle light-scattering apparatus under shear flow (Rheo-SALS), which enables us to perform time-resolved measurements of light intensity scattered from the internal structure developed under shear flow [38]. They examined long-range correlation and its evolution in the organo-hectorite/styrene $(3.5 / 96.5 \mathrm{vol} / \mathrm{vol})$ suspensions (dense condition: volume fraction $\phi \geq 0.01$ [39]) under shear flow. In this region, the power law relation for both zero- $\omega$ limiting value of modulus $G^{\prime}(\omega \rightarrow 0)$ and fluidizing stress $\left(\sigma_{\mathrm{fl}}\right)$ were given by

$$
G^{\prime}(\omega \rightarrow 0) \sim \sigma_{f l} \propto \varphi^{4 \pm 0.2}
$$

The ratio of $G^{\prime \prime}(\omega) / G^{\prime}(\omega)(=\tan \delta)$ exhibits independence of $\phi$ and remains a constant value of about 0.1 . This behavior relates to self-similarity of the linked network structure in the gelation region as reported by Winter [40].

The hectorite $\left(\mathrm{Na}_{0.33}\left(\mathrm{Mg}_{2.67} \mathrm{Li}_{0.33}\right) \mathrm{Si}_{4} \mathrm{O}_{10}(\mathrm{OH})_{2}\right)$ was modified with quarternized hexadecyl, octadecyl ammonium chloride $\left(\left[\left(\mathrm{C}_{16} \mathrm{H}_{33}\right)_{0.5}\left(\mathrm{C}_{18} \mathrm{H}_{37}\right)_{1.5} \mathrm{~N}^{+}(\mathrm{C}\right.\right.$ $\left.\left.\left.\mathrm{H}_{3}\right)_{2}\right] \mathrm{Cl}^{-}\right)$[38]. The estimated individual silicate layer under very dilute conditions $(\phi<<0.01)$ was $60 \mathrm{~nm}$ in length scale. Four different $\dot{\gamma}$ were selected to confirm the time dependence of the internal structural change under different steady shear flow via stress rheometer. The time development of transient viscosity $(\eta(\dot{\gamma}: t))$ under constant shear rate $(\dot{\gamma})$ for the first run at $25^{\circ} \mathrm{C}$ is shown in Figure 3.6. Under $\dot{\gamma}=0.1$ and $1.0 \mathrm{~s}^{-1}, \eta(\dot{\gamma}: t)$ gradually increases with time and exhibits the power law, $\eta(\dot{\gamma}: t) \propto t^{0}$ with $v=0.5$ for $0.1 \mathrm{~s}^{-1}$ and $v=0.2$ for $1.0 \mathrm{~s}^{-1}$, whereas under $\dot{\gamma}=10$ and $10^{2} \mathrm{~s}^{-1}$ (yielding regime due to the strong shear stress), $\eta(\dot{\gamma}: t)$ decreases, and power law decay, $\eta(\dot{\gamma}: t) \propto t^{-v}$ with $v=0.17$ is exhibited for both cases (Figure 3.6). The quite different viscosity profiles under constant $\dot{\gamma}$ runs were reported.

The time variation of the mean-square density fluctuation $\left\langle\eta^{2}\right\rangle$, the mean-square anisotropy $\left\langle\delta^{2}\right\rangle$, and the relevant value of long-range correlation distance $\left(\xi_{\eta}\right.$ and $\xi_{\delta}$ ) upon imposition/cessation of steady shear flow at both low $\left(=0.5 \mathrm{~s}^{-1}\right)$ and high $\dot{\gamma}\left(=60 \mathrm{~s}^{-1}\right)$ were discussed (Figure 3.7). Before flowing, the organo-clay network in length scale was $450 \mathrm{~nm}$ estimated from $\xi_{\eta}$ value, suggesting the existence of a large-scale flocculated network structure due to the edge-to-edge interaction [41]. In addition, the boundary between networks is of importance in predicting the complex flow dynamics. The suppression of $\left\langle\eta^{2}\right\rangle$ is observed during flow 


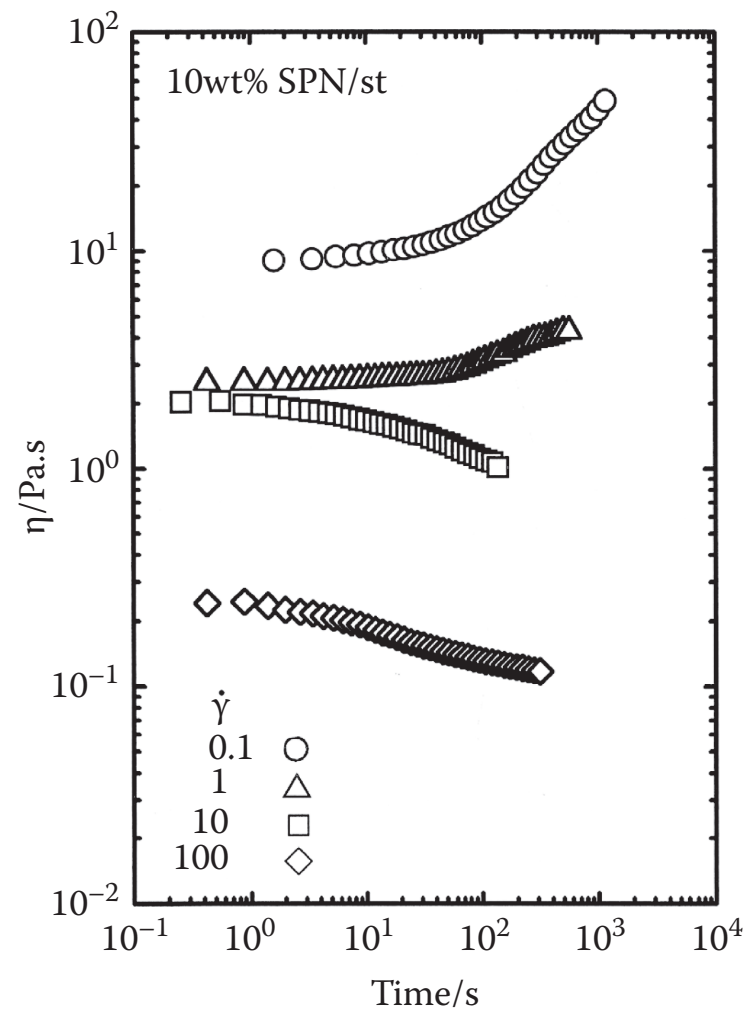

FIGURE 3.6

Time variation of shear viscosity for organo-hectorite/styrene (3.5/96.5 vol/vol) suspension with four shear rate. (Reprinted from Okamoto M, Sato H, Taguchi H, Kotaka T, Nippon Rheology Gakkaishi 28, 201.Copyright 2000 The Society of Rheology, Japan. With permission.)

in both cases as compared with the initial quiescent state (before shearing). Presumably, this is due to the hydrodynamic forces, which promote alignment of the organo-clay networks because of the large dimensions of the networks.

Under $\dot{\gamma}=0.5 \mathrm{~s}^{-1}$ (corresponding to unabated viscosity regime), the gradual decreasing of $\left\langle\eta^{2}\right\rangle$ and increasing of $\xi_{\eta}$ upon imposition of shear are observed. Simultaneously, the value of $\left\langle\delta^{2}\right\rangle$ is jumped upward and then remains almost constant during flow, where $\xi_{\delta}$ also keeps a constant value of $400 \mathrm{~nm}$, indicating that the anisotropy in the system is developed. Upon cessation of shear at $750 \mathrm{~s}$, on the contrary, $\left\langle\eta^{2}\right\rangle$ increases with time and finally levels off at $1300 \mathrm{~s}$ with $\xi_{\eta}$ of $250 \mathrm{~nm}$. On the other hand, $\left\langle\delta^{2}\right\rangle$ does not become zero and developed orientation of the network with $\xi_{\delta}$ of $690 \mathrm{~nm}$ in the shear field is stable, even upon cessation of shear. The anisotropy gradually develops with time, forming relatively stable oriented networks, which are stable even after the cessation of the shear. 


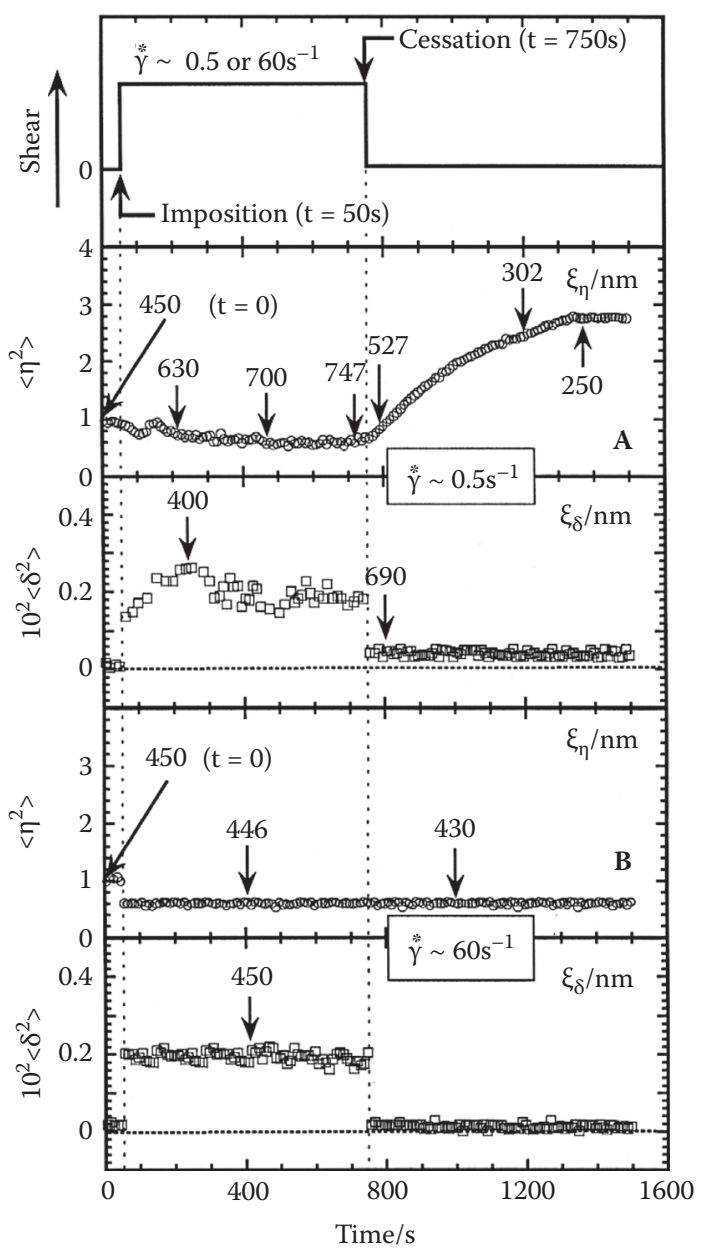

\section{FIGURE 3.7}

Time variation of $\left\langle{ }_{-}^{2}\right\rangle$ and $\left\langle-_{-}{ }^{2}\right\rangle$ upon imposition/cessation of steady shear under low $(=0.5$ $\left.\mathrm{s}^{-1}\right)$ and high $\dot{\gamma}\left(=60 \mathrm{~s}^{-1}\right)$ conditions. The Debye-Bueche equation is applicable in anisotropic AU: Correct spelling? shear flow field for dence suspension [42]. (Reprinted from Okamoto M, Sato H, Taguchi H, Kotaka T, Nippon Rheology Gakkaishi 28, 201.Copyright 2000 The Society of Rheology, Japan. With permission.)

In the case of under $\dot{\gamma}=60 \mathrm{~s}^{-1},\left\langle\eta^{2}\right\rangle$ slightly decreases upon imposition of shear, but $\xi_{\eta}$ does not change and agrees with the value of the initial quiescent state during flow. For the anisotropy, during shear flow, $\left\langle\delta^{2}\right\rangle$ stably appears with $\xi_{\delta}$ of $450 \mathrm{~nm}$; upon cessation of shear, it disappears suddenly, suggesting that such an aligned, orientated network structure in the high shear field is labile (not stable) as compared with that under weak shear flow. Furthermore, following the large deformation, the slippage and rotation take 
place at the interface between network domains, and the resulting shear thinning behavior is observed in this condition [39]. This feature may resemble the rotation of the grains in block copolymer melt under uniaxial deformation [43]. In PCN melts, however, the network structure is easily destroyed (ruptured) by deformation due to the polymer chain entanglement as discussed by Solomon et al. [19].

The structure disorder creates energy barriers that prevent reorganizing/ reconstituting networks into states of lower free energy. In dynamics for a typical soft glassy material, a slow degree of freedom is taken into account $[12,44]$. Jamming is a common property of complex fluids. In the presence of stress, the viscosity is given by the distribution of relaxation times of the networks of "slow mode."

Imposition of shear is considered to change the energy landscape and allows for the system to access new metastable states [45]. For this reason, the imposition of weak shear $\left(\dot{\gamma}=0.5 \mathrm{~s}^{-1}\right)$ (much smaller than yield stress) is considered as a shear rejuvenating condition $\left(\eta(\dot{\gamma}: t) \propto t^{0.5}\right)$, meaning that the longest relaxation time of the slow mode decreases in time. Accordingly, the initial network structure grows with time, accompanied by an increase of $\xi_{\text {n }}$.

Upon cessation of shear, aging begins anew, because flow alters the energy landscape, new metastable states are now accessible, and the system evolves spontaneously, accompanied by an increase of $\xi_{\delta}$ and decrease of $\xi_{\eta}$. This suggests that aging of the system (i.e., the reconstituting in between networks) starts, and a steady state is finally reached.

In the case of large deformation $\left(\dot{\gamma}=60 \mathrm{~s}^{-1}\right)$, those energy barriers become greater the longer a system is aged, such that the longest relaxation time continuously increases. As a result, upon cessation of shear, thermal motion alone is insufficient to mediate complete structural relaxation. Therefore, the network may become trapped in a higher energy state (almost constant value of $\xi_{\eta}$ ). Brownian forces alone are unable to change the energy barriers created by such an oriented organo-clay network structure, because the estimated rotational Brownian motion of the hectrite platelets is about $10^{-2} \mathrm{~s}$ at $25^{\circ} \mathrm{C}$. Such discussion on energy landscape appears to be entirely valid for the experimental results as well as isotropic particle dispersion in a Newtonian fluid matrix [44].

\subsection{Alignment of Silicate Layers in Networks}

The organo-clay platelets orient in both shear and elongational flow fields. A second question is how the platelets are oriented during the flow direction. Lele et al. [46] reported the in situ Rheo-X-ray investigation of flow-induced orientation in syndiotactic PP/layered silicate nanocomposite melt. The clay 
platelets rapidly oriented and remain at constant orientation in the long time regime ( $1500 \mathrm{~s})$

The orientation of silicate layers and nylon 6-base nanocomposite (N6CN) using ex situ small-angle X-ray scattering (SAXS) is examined [33]. The clay layers, due to their higher aspect ratio, were predominantly oriented in a "parallel" orientation (with layer normals along the velocity gradient direction) at different times following LAOS.

Kojima et al. [47] found three regions of different orientations in the injection-molded bar as a function of depth. Near the middle of the sample, where the shear forces are minimal, the clay platelets are oriented randomly, and the nylon 6 crystallites are perpendicular to the silicate layers. In the surface region, shear stresses are very high, so both the clay layers and the nylon 6 crystallites are parallel to the surface. In the intermediate region, the clay layers, presumably due to their higher aspect ratio, still orient parallel to the surface, and the nylon 6 crystallites assume an orientation perpendicular to the silicate. Medellin-Rodriguez et al. [48] reported that the molten N6CN samples showed planar orientation of silicate layers along the flow direction, which is strongly dependent on shear time as well as clay loading, reaching a maximally orienting level after being sheared for 15 min with $\dot{\gamma}=60 \mathrm{~s}^{-1}$.

In contrast, the orientation occurs by the "normal" to the clay surface aligning the flow direction through vorticity during shear. Okamoto et al. conducted the transmission electron microscopic (TEM) observation for the sheared N6CN3.7 (clay loading $=3.7 \mathrm{wt} \%$ ) with $\dot{\gamma}=0.0006 \mathrm{~s}^{-1}$ for $1000 \mathrm{~s}$ [49]. The edges of the silicate layers laying along the z-axis (marked with the arrows (A)) or parallel alignment of the silicate edges to the shear direction ( $x$-axis) (marked with the arrows (B)) rather than random orientation in the matrix is observed, but in fact, one cannot see these faces in this plane (Figure 3.8). Here, it should be emphasized that the planar orientation of the silicate faces along the $x-z$ plane does not take place prominently. For the case of rapid shear flow, the commonly applicable conjecture of the planar orientation of the silicate faces along the shear direction first demonstrated to be true by Kojima et al. [47].

In uniaxial elongational flow (converging low) for a PP-based nanocomposite (PPCN4) (clay loading $=4 \mathrm{wt} \%$ ), the formation of a house-of-cards structure is found by TEM analysis [50,51]. The perpendicular (not parallel) alignment of clay platelets with large anisotropy toward the flow direction might sound unlikely, but this could be the case, especially under an elongational flow field, in which the extentional flow rate is the square of the converging flow rate along the thickness direction, if the assumption of affine deformation without volume change is valid. Obviously, under such conditions, the energy dissipation rate due to viscous resistance between the platelet surface and the matrix polymer is minimal when the platelets are aligned perpendicular to the flow direction. 


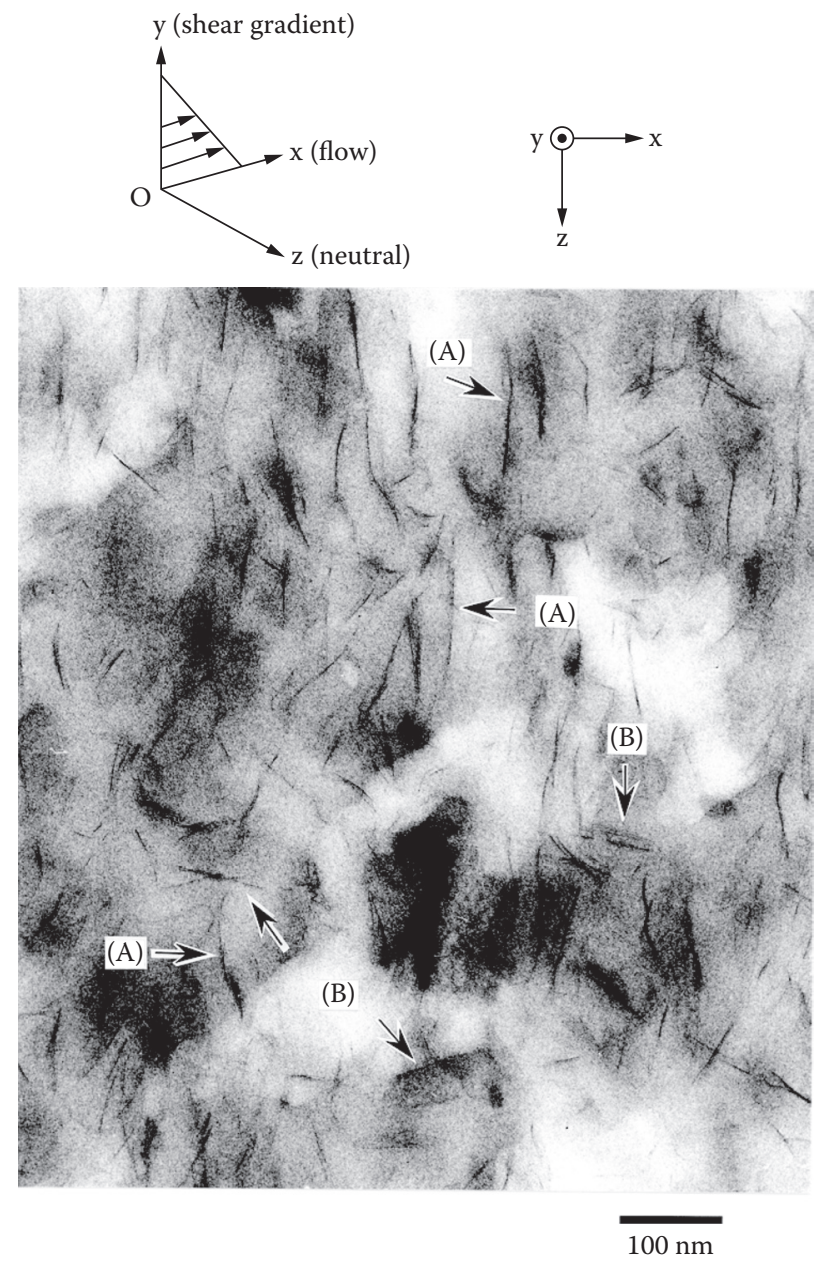

FIGURE 3.8

Transmission electron micrograph (TEM) in the $x-z$ plane showing N6CN (clay loading $=3.7$ $\mathrm{wt} \%$ ) sheared at $225^{\circ} \mathrm{C}$ with $\dot{\gamma}=0.0006 \mathrm{~s}^{-1}$ for $1000 \mathrm{~s}$. The $x-, y-$, and $z$-axes correspond respectively to flow, shear gradient, and neutral direction. (Reprinted from Okamoto M, Rapra Review Report 163. Copyright 2003 Rapra Technology Ltd., London. With permission.)

Figure 3.9 shows double logarithmic plots of transient elongational viscosity $\eta_{E}\left(\dot{\varepsilon}_{0} ; t\right)$ against time $t$ observed for N6CN3.7 and PPCN4 with different Hencky strain rates $\dot{\varepsilon}_{0}$ ranging from $0.001 \mathrm{~s}^{-1}$ to $1.0 \mathrm{~s}^{-1}$. The solid curve represents time development of threefold shear viscosity, $3 \eta_{0}(\dot{\gamma} ; t)$, at $225^{\circ} \mathrm{C}$ with a constant shear rate $\left(\dot{\gamma}=0.001 \mathrm{~s}^{-1}\right)$. First, the extended Trouton rule, $3 \eta_{0}(\dot{\gamma} ; t) \cong \eta_{E}\left(\dot{\varepsilon}_{0} ; t\right)$ [52], as well as an empirical Cox-Merz relation ( $\left.\eta(\dot{\gamma})=\left|\eta^{*}\right|(\omega)\right)$ [53], fails for both N6CN3.7 and PPCN4 melts, as opposed to the melt of ordinary homopolymers. In $\eta_{E}\left(\dot{\varepsilon}_{0} ; t\right)$ at any $\dot{\varepsilon}_{0}$, the N6CN3.7 melt shows a weak tendency of strain-induced hardening as compared to that 


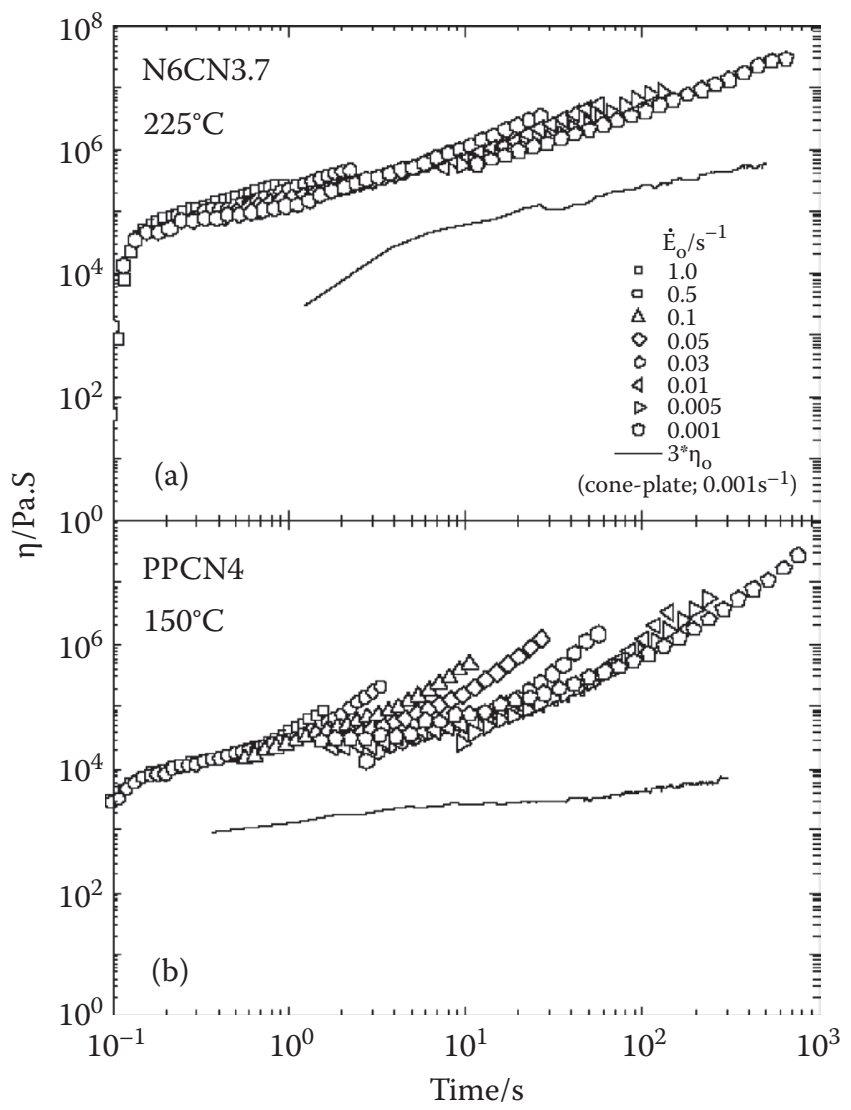

FIGURE 3.9

Time variation of elongational viscosity $\eta_{E}\left(\dot{\varepsilon}_{0} ; t\right)$ for (a) N6CN3.7 melt at $225^{\circ} \mathrm{C}$ and for (b) PPCN4 at $150^{\circ} \mathrm{C}$. The solid line shows three times the shear viscosity, $3 \eta_{E}(\dot{\gamma} ; t)$, taken at a low shear rate $\dot{\gamma}=0.001 \mathrm{~s}^{-1}$ on a cone-plate rheometer. (Reprinted from Okamoto M, Rapra Reviezw Report 163. Copyright 2003 Rapra Technology Ltd., London; Okamoto M, Nam PH, Maiti P, Kotaka T, Hasegawa N, Usuki A, Nano Lett. 1, 295, 2001. With permission.)

of the PPCN4 melt. A strong behavior of strain-induced hardening for the PPCN4 melt was originated from an aging phenomenon characteristic of reconstituting the networks through the perpendicular alignment of the silicate platelets to the stretching direction.

From TEM observation (see Figure 3.8), the N6CN3.7 forms a fine dispersion of the silicate platelets of about $100 \mathrm{~nm}$ in $L_{\text {clay, }} 3 \mathrm{~nm}$ thickness in $d_{\text {clay }}$ and _clay of about 20 to $30 \mathrm{~nm}$ between them. The _clay value is one order of magnitude lower than the value of $L_{\text {clay }}$ suggesting the formation of a rigid network domain structure of the dispersed clay platelets in end-tethered polymer chains (see flow activation energy in Figure 3.10). This suggests that both slow $\left(\dot{\gamma}=0.001 \mathrm{~s}^{-1}\right)$ and rapid $\left(\dot{\gamma}=1.0 \mathrm{~s}^{-1}\right)$ elongational flow rates are 


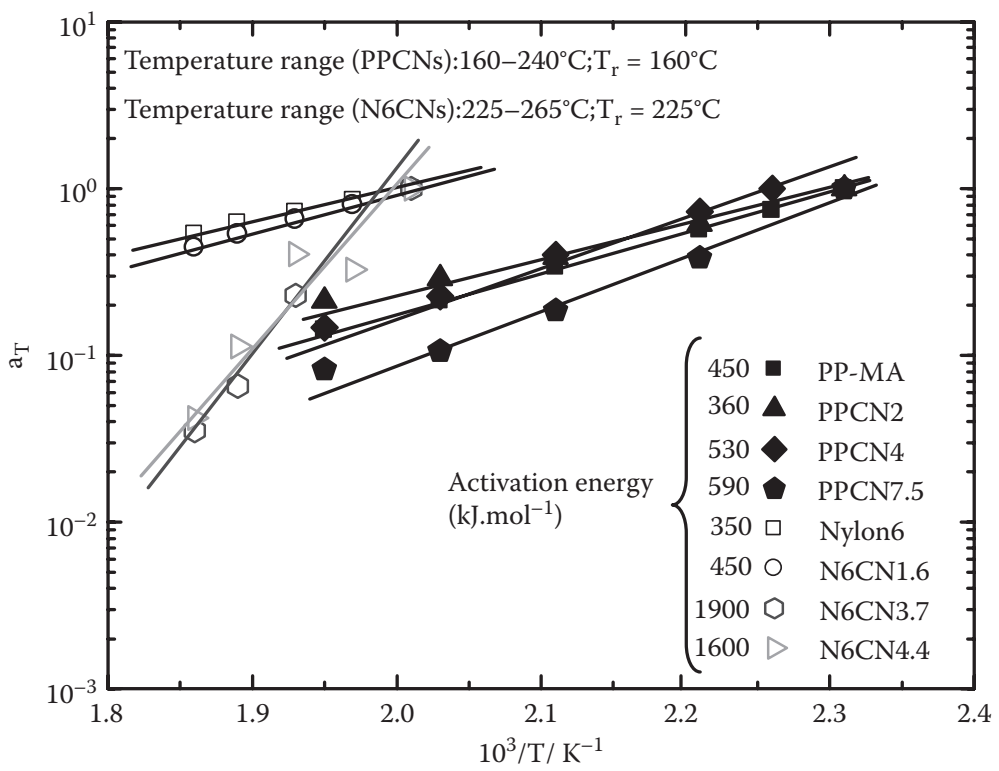

FIGURE 3.10

Frequency shift factors $a_{\mathrm{T}}$ as a function of temperature. Flow activation energy of neat PP and nylon 6, PPCNs and N6CNs with different contents of clay are shown.

unable to erase the energy barriers created by the in situ polymerization condition. Accordingly, the longest relaxation time in the network may remain constant. This tendency was also observed in the PPCN7.5 melt having a higher content of clay $(=7.5 \mathrm{wt} \%)$ [31].

SANS is useful in determining the orientation of the organo-clay under shear because of contrast matching to clay (montmorillonite) in $\mathrm{D}_{2} \mathrm{O}$ [34]. In aqueous dispersions of hectrite ( $3 \mathrm{wt} \%$ ) and poly(ethylene oxide) (PEO) (2 $\mathrm{wt} \%$ ), the platelets were oriented in the flow direction with the surface normal in the neutral direction (Figure 3.11) [34]. It is quite possible that the dispersed organo-clay platelets attain not only parallel alignment but also perpendicular or even transverse alignment during shear and elongational flow fields.

The aqueous clay (kaolinite) suspensions have been investigated both in the quiescent state [54] and under shear flow using SANS [55]. Some 20 years ago, van Olphen [51] pointed out that the electrostatic attraction between the layers of natural clay in aqueous suspension arises from higher polar force in the medium. The intriguing features such as yield stress thixotropy and rheopexy exhibited in aqueous suspensions of natural clay minerals may be taken as a reference to the present PCNs. More detailed surveys on various types of experiments can also be found in the literature [56-63]. 


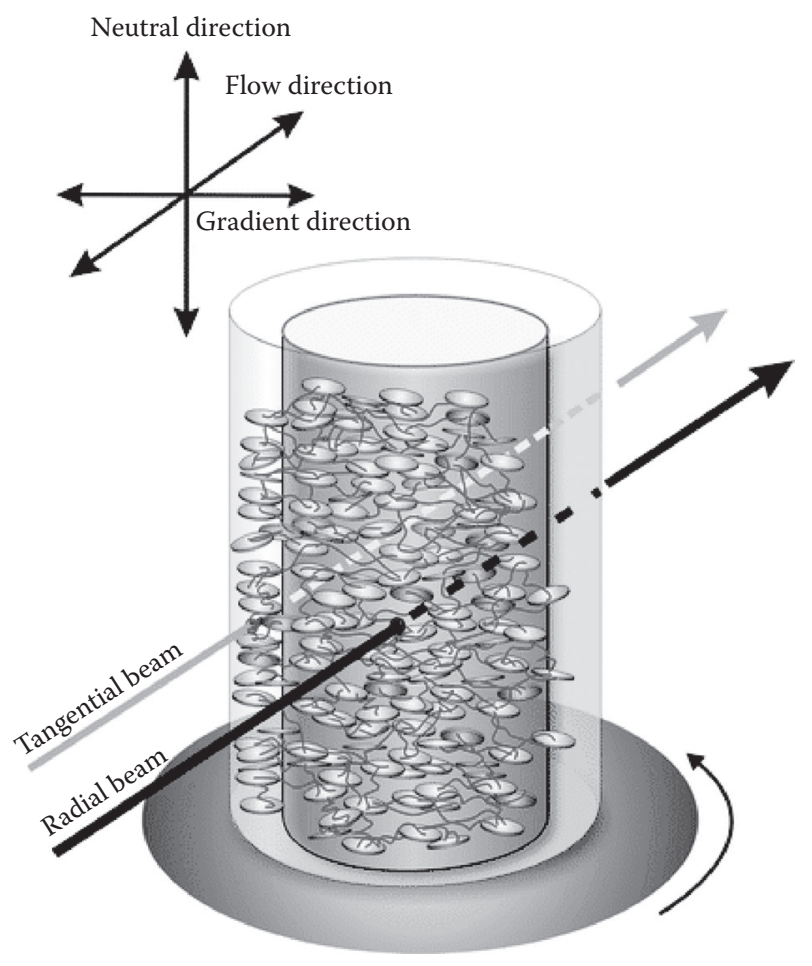

FIGURE 3.11

Couette-type shear cell for small-angle neutron scattering (SANS) and model for real space orientation of oriented clay platelets in the cell. The reference coordinate frame is anchored in the tangential beam. (Reprinted from Scmidt G, Nakatani AI, Butler PD, Karim A, Han CC, Macromolecules 33, 7219.Copyright 2000 American Chemical Society. With permission.

\subsection{Summary}

For an improved understanding of the soft glassy dynamics in PCN melts, in this review, we described some recent results concerning the mesoscale organo-clay network structure and its reversibility in the light of melt rheometry with a combination of scattering experiments and electron microscopy. We studied the dynamics in PCN melts; however, it is difficult to discuss the intrinsic feature of the networks.

Although our experimental results are still weak evidence to discuss the mesoscale structure development in PCN, many papers ignore the existence of percolated organo-clay networks and their intrinsically metastable states and out-of-equilibrium condition. Once a percolated network is formed, the networks retard crystalline capability and enhance thermal stability as well as modulus $[64,65]$. 
For the future, for designing high-performance PCN materials and their processing, the correlation between the mesoscale network structure and macroscopic properties will be probed via an innovative methodology such as three-dimensional TEM and fast scanning Fourier transform infrared (FTIR) imaging.

\section{References}

1. Sinha Ray S, Okamoto M, "Polymer/Layered Silicate Nanocomposites: A Review from Preparation to Processing" Prog. Polym. Sci., 28, 1539-1641 (2003).

2. Vaia RA, Wagner HD, "Framework for Nanocomposites" Materials Today, 7, 32-37 (2004).

3. Gao F, "Clay/Polymer Composites: The Story" Materials Today, 7, 50-55 (2004).

4. Okamoto M, "Recent Advances in Polymer/Layered Silicate Nanocomposites: An Overview from Science to Technology" Mater. Sci. Tech., 22, 7, 756-779 (2006).

5. Okada A, Usuki A, "Twenty Years of Polymer-Clay Nanocomposites" Macromol. Mater. Eng., 291, 1449-1476 (2006).

6. Hussain F, Hojjati M, Okamoto M, Gorga RE, "Review Paper: Polymer-Matrix Nanocomposites, Processing, Manufacturing, and Application: An Overview" J. Composite Mater., 40, 1511-1575 (2006).

7. Usuki A, Kojima Y, Okada A, Fukushima Y, Kurauchi T, Kamigaito O, J. Mater. Res., 8, 1174 (1993).

8. Vaia RA, Giannelis EP, Macromolecules, 30, 8000 (1997).

9. Krishnamoorti R, Vaia RA, Giannelis EP, Chem. Mater., 8, 1728 (1996).

10. Rao Y, Pochan JM, Macromolecules, 40, 290 (2007).

11. Treece MA, Oberhauser JP, Macromolecules, 40, 571 (2007).

12. Sollich P, Lequeux F, Hebraud P, Cate ME, Phys. Rev. Lett., 78, 2020 (1997).

13. Krishnamoorti R, Giannelis EP, Macromolecules, 30, 4097 (1997).

14. Fornes TD, Yoon PJ, Keskkula H, Paul DR, Polymer, 42, 9929 (2001).

15. Hoffman B, Dietrich C, Thomann R, Friedrich C, Mulhaupt R, Macromol. Rapid Commun., 21, 57 (2000).

16. Ren J, Silva AS, Krishnamoorti R, Macromolecules, 33, 3739 (2000).

17. Mitchell CA, Krishnamoorti R, J. Polym. Sci. Part B Polym. Phys., 40, 1434 (2002).

18. Lepoittevin B, Devalckenaere M, Pantoustier N, Alexandre M, Kubies D, Calberg C, Jerome R, Dubois P, Polymer, 43, 1111 (2002).

19. Solomon MJ, Almusallam AS, Seefeld KF, Somwangthanaroj S, Varadan $P$, Macromolecules, 34, 1864 (2001).

20. Galgali G, Ramesh C, Lele A, Macromolecules, 34, 852 (2001).

21. Lele A, Mackley M, Galgali G, Ramesh C, J. Rheol., 46, 1091 (2002).

22. Treece MA, Oberhauser JP, Polymer, 48, 1083 (2007).

23. Sinha Ray, S, Maiti, P, Okamoto, M, Yamada, K, Ueda, K, Macromolecules, 35, 3104 (2002).

24. Sinha Ray S, Yamada K, Okamoto M, Ueda K, Polymer, 44, 6631 (2003).

25. Sinha Ray S, Okamoto K, Okamoto M, Macromolecules, 36, 2355 (2003). 
26. Okamoto K, Sinha Ray S, Okamoto M, J. Polym. Sci. Part B: Polym. Phys., 41B, 3160 (2003).

27. Krishnamoorti R, Yurekli K, Current Opinion in Colloid Interface Sci. 6, 464 (2001).

28. Maiti P, Nam PH, Okamoto M, Kotaka T, Hasegawa N, Usuki A, Macromolecules, 35, 2042 (2002).

29. Williams ML, Landel RF, Ferry JD, J. Amer. Chem. Soc., 77, 3701 (1955).

30. Maiti P, Okamoto M, Macromole. Mater. Eng., 288, 440 (2003).

31. Nam, PH, Master Thesis, Toyota Technological Institute (2001).

32. Brenner H, Int. J. Multiphase Flow, 1, 195 (1974).

33. Ren J, Casanueva BF, Mitchell CA, Krishnamoorti R, Macromolecules, 36, 4188 (2003).

34. Scmidt G, Nakatani AI, Butler PD, Karim A, Han CC, Macromolecules, 33, 7219 (2000).

35. Saito T, Okamoto M, Hiroi R, Yamamoto M, Shiroi T, Polymer, 48, 4143 (2007).

36. Tanaka H, Meunier J, Bonn D, Phys Rev. E, 69, 031404 (2004).

37. Mourchid A, Delville A, Lambard J, Lecolier E, Levitz P, Langmuir, 11, 1942 (1995).

38. Okamoto M, Sato H, Taguchi H, Kotaka T, Nippon Rheology Gakkaishi, 28, 201 (2000).

39. Okamoto M, Taguchi H, Sato H, Kotaka T, Tatayama H, Langmuir, 16, 4055 (2000).

40. Winter HH, Chambon F, J. Rheol., 30, 367 (1986).

41. Sinha Ray S, Okamoto K, Okamoto M, Macromolecules, 36, 2355 (2003).

42. Hsiao BS, Stein RS, Deutscher K, Winter HH, J. Polym. Phys., 28, 1571 (1990).

43. Kobori Y, Kwon YK, Okamoto M, Kotaka T, Macromolecules, 36, 1656 (2003).

44. Bonn D, Tanase S, Abou B, Tanaka H, Meunier J, Phys. Rev. Lett., 89, 015701 (1992).

45. Lacks D, Phys Rev. E, 64, 51508 (2001).

46. Lele A, Mackley M, Galgali G, Ramesh C, J. Rheol., 46, 1091 (2002).

47. Kojima Y, Usuki A, Kawasumi M, Okada A, Kurauchi T, Kamigaito O, Kaji K, J. Polym. Sci. Part B: Polym. Phys., 33, 1039 (1995).

48. Medellin-Rodriguez FJ, Burger C, Hsiao BS, Chu B, Vaia RA, Phillips S, Polymer, 42, 9015 (2001).

49. Okamoto M, "Polymer/Layered Silicate Nanocomposites," Rapra Review Report 163, 166 pp, Rapra Technology Ltd., London (2003).

50. Okamoto M, Nam PH, Maiti P, Kotaka T, Hasegawa N, Usuki A, Nano. Lett., 1, 295 (2001).

51. van Olphen H, An Introduction to Clay Colloid Chemistry, Wiley: New York (1977).

52. Trouton FT, Proc. Roy. Soc., A77, 426 (1906).

53. Cox WP, Merz EH, J. Polym. Sci., 28, 619 (1958).

54. Pignon F, Magnin A, Piau JM, J. Rheol., 42, 1349 (1998).

55. Jogun SM, Zukoski CF, J. Rheol., 43, 847 (1999).

56. Roe R, Methods of X-ray and Neutron Scattering in Polymer Science. New York: Oxford University Press, p.199 (2000).

57. Bafna A, Beaucage G, Mirabella F, Skillas G, Sukumaran S, J. Polym. Sci., Part B: Polym. Phys., 39, 2923 (2001).

58. Koo CM, Kim SO, Chung IJ, Macromolecules, 36, 2748 (2003). 
59. Yalcin B, Valladares D, Cakmak M, Polymer, 44, 6913 (2003).

60. Bafna A, Beaucage G, Mirabella F, Mehta S, Polymer, 44, 1103 (2003).

61. Yalcin B, Cakmak M, Polymer, 45, 2691 (2004).

62. Loo LS, Gleason KK, Polymer, 45, 5933 (2004).

63. Kim JH, Koo CM, Choi YS, Wang KH, Chung IJ, Polymer, 45, 7719 (2004).

64. Wang K, Liang S, Deng J, Yang H, Zhang Q, Fu Q, Dong X, Wang D, Han CC, Polymer, 47, 7131 (2006).

65. Rao Y, Pochan JM, Macromolecules, 40, 290 (2007). 